\title{
Interactive comment on "Stratospheric influence on MLT over mid-latitudes in winter by Fabry-Perot interferometer data" by Olga S. Zorkaltseva and Roman V. Vasilyev
}

Olga S. Zorkaltseva and Roman V. Vasilyev

meteorologist-ka@yandex.ru

Received and published: 15 December 2020

I and the co-author would like to thank the Referee for the comments. 1. Indeed, we have chosen the term "stationary planetary wave" incorrectly. We will replace this term with "planetary wave". Note that in the preliminary study, we considered the phase of the planetary wave, but did not find a significant relationship between the phase and processes in MLT. Most often, the wave is located to the east of our observatory. 2. We call the 2019-2020 SSW atypical because the warming started one month later than usual. We wanted to emphasize the atypically late time of the SSW occurrence. 
referee, in section 3.2 we will discuss each SSW and PW case in more detail. Note that the periods of missing observations may contain not very correct data. As, for example, at the beginning of SSW1 in 2016-2017. Perhaps we will add a graph of the number of measurements during the day to each figure. And those days when there are few measurements we will consider with caution. I agree that there are questions with temperature variations, but oxygen emission always decreases with an active stratosphere. 4. Line 183. Absolutely. We'll fix it. 5. Figure 16. Ok, we'll add a legend. 6. Line 227. This is a typo, right - SSW. 7. Section 3.4, lines 229-230. Thanks for the recommendation, we will add interpretation to section 3.4.

Interactive comment on Ann. Geophys. Discuss., https://doi.org/10.5194/angeo-2020-73, 2020. 